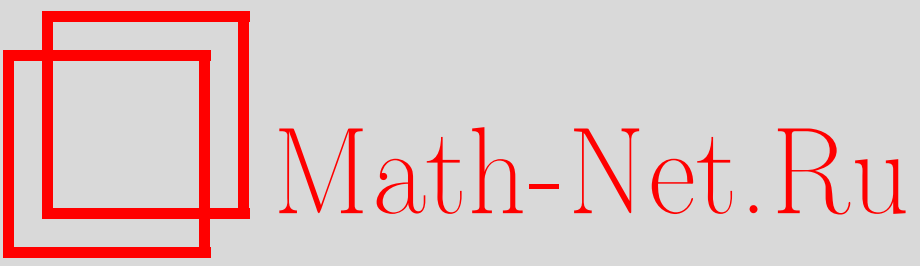

М. В. Булычева, О сходимости интервалов справедливых цен в модели с Европейскими опционами, УМН, 2005, том 60, выпуск 2, 149-150

DOI: https://doi.org/10.4213/rm1404

Использование Общероссийского математического портала Math-Net.Ru подразумевает, что вы прочитали и согласны с пользовательским соглашением

http://www . mathnet.ru/rus/agreement

Параметры загрузки:

IP: 54.166 .219 .16

26 апреля 2023 г., 17:15:27 


\title{
О СХОДИМОСТИ ИНТЕРВАЛОВ СПРАВЕДЛИВЫХ ЦЕН В МОДЕЛИ С ЕВРОПЕЙСКИМИ ОПЦИОНАМИ
}

\author{
М. В. БУЛЫчЕВА
}

Одна из популярных в современной финансовой математике моделей основана на рассмотрении всевозможных опционов-колл Европейского типа на некоторьй актив с фиксированной датой исполнения. Более точно, пусть $S_{T}$ - положительная случайная величина на вероятностном пространстве $(\Omega, \mathscr{F}, \mathrm{P})$, имеющая смысл цены некоторого финансового актива в момент времени $T$; $\mathbb{K} \subseteq \mathbb{R}_{+}-$множество цен исполнения имеющихся на рынке опционов-колл Европейского типа на этот актив (владелец опциона получает сумму $\left(S_{T}-K\right)^{+}$в момент $\left.T\right) ; \varphi(K), K \in \mathbb{K},-$ цена в момент 0 такого опциона (для простоты предполагаем, что безрисковая процентная ставка равна нулю). Эта модель была впервые рассмотрена в работах [1], [2]. Обзор литературы по данной модели можно найти в [3].

В настоящей работе данная модель рассматривается с точки зрения подхода, предложенного в [4]. Именно, в этой работе вводится условие отсутствия обобщенного арбитража (это усиление обычного понятия безарбитражности; последнее является слишком слабым в моделях с бесконечным числом активов, в частности, в нашей модели) и доказьвается, что при $\mathbb{K}=\mathbb{R}_{+}$модель удовлетворяет этому условию, если и только если

(a) $\varphi$ вьпукла на $\mathbb{R}_{+}$;

(b) $\varphi_{+}^{\prime}(0) \geqslant-1$;

(c) $\lim _{K \rightarrow \infty} \varphi(K)=0$;

(d) $\varphi^{\prime \prime} \sim \operatorname{Law} S_{T}$

где $\varphi_{+}^{\prime}$ обозначает правостороннюю производную, а $\varphi^{\prime \prime}$ обозначает вторую производную в смысле распределений (т.е. $\varphi^{\prime \prime}$ - это мера, определяемая формулой $\left.\varphi^{\prime \prime}((a, b])=\varphi_{+}^{\prime}(b)-\varphi_{+}^{\prime}(a)\right)$ с соглашением $\varphi^{\prime \prime}(\{0\})=\varphi_{+}^{\prime}(0)+1$, а знак “ ", как обьчно, обозначает эквивалентность мер.

Рассмотрим теперь платежное поручение $F=f\left(S_{T}\right)$, где $f: \mathbb{R}_{+} \rightarrow \mathbb{R}$ - измеримая функция. В [4] определяется множество справедливых цен $I(F)$ платежного поручения $F$ и доказьвается, что если $\mathbb{K}=\mathbb{R}_{+}$, модель удовлетворяет условию отсутствия обобщенного арбитража и функция $f$ ограничена снизу, то

$$
I(F)= \begin{cases}\left\{\int_{\mathbb{R}_{+}} f(x) \varphi^{\prime \prime}(d x)\right\}, & \text { если } \int_{\mathbb{R}_{+}} f(x) \varphi^{\prime \prime}(d x)<\infty, \\ \varnothing & \text { иначе. }\end{cases}
$$

Предположение $\mathbb{K}=\mathbb{R}_{+}$очень удобно с теоретической точки зрения, поскольку оно приводит к одноточечному множеству справедливых цен, но на практике $\mathbb{K}$ конечно (обычно оно состоит из 10-20 точек). В случае конечного $\mathbb{K}$ условие отсутствия обобщенного арбитража равносильно обьчному условию безарбитражности, состоящему в том, что $A \cap L_{+}^{0}=\{0\}$, где $L_{+}^{0}$ обозначает множество неотрицателшных случайных величин на $(\Omega, \mathscr{F}, \mathrm{P})$, а

$$
A=\left\{\sum_{m=1}^{M} h_{m}\left(\left(S_{T}-K_{m}\right)^{+}-\varphi\left(K_{m}\right)\right): M \in \mathbb{N}, h_{m} \in \mathbb{R}, K_{m} \in \mathbb{K}\right\}
$$

(с финансовой точки зрения, $A$ - множество возможных прибылей, которые можно получить в данной модели). Далее, в случае конечного $\mathbb{K}$ множество $I(F)$ является интервалом с концевыми точками $C_{*}(F)$ и $C^{*}(F)$, где

$$
\begin{aligned}
& C_{*}(F)=\sup \{x: \text { существует } X \in A \text { такой, что } x-X \leqslant F \text { Р-п.н. }\}, \\
& C^{*}(F)=\inf \{x: \text { существует } X \in A \text { такой, что } x+X \geqslant F \text { Р-п.н. }\} .
\end{aligned}
$$

Возникает следующий естественньй вопрос. Предположим, что $\left(\mathbb{K}_{n}\right)_{n=1}^{\infty}-$ последовательность конечных подмножеств $\mathbb{R}_{+}$, “сходящаяся" $\kappa \mathbb{R}_{+}$. При каких условиях на 
$F=f\left(S_{T}\right)$ имеет место сходимость $I_{n}(F) \underset{n \rightarrow \infty}{\longrightarrow} I(F)$ ? Здесь $I_{n}(F)$ - интервал справедливых цен $F$ в модели, основанной на $\mathbb{K}_{n}$, а $I(F)$ - интервал справедливых цен $F$ в модели, основанной на $\mathbb{R}_{+}$, т.е. $I(F)$ задается формулой $(1)$. При этом имеется в виду, что все "допредельные" модели и "предельная" модель заданы на одном и том же пространстве $(\Omega, \mathscr{F}, \mathrm{P})$ с одной и той случайной величиной $S_{T}$ и основаны на одной и той же функции $\varphi: \mathbb{R}_{+} \rightarrow \mathbb{R}_{+}$(так что в "допредельных" моделях используются ее сужения на $\left.\mathbb{K}_{n}\right)$. Этот вопрос имеет некоторое сходство с проблемами теории "больших" рынков (см. [5; гл. VI]), но существенное отличие от этой теории состоит в том, что у нас имеется "предельная" модель.

Прежде всего, необходимо конкретизировать сходимость $\mathbb{K}_{n} \underset{n \rightarrow \infty}{\longrightarrow} \mathbb{R}_{+}$. Естественно понимать эту сходимость в следующем смысле:

$$
\forall x \in \mathbb{R}_{+}, \forall \varepsilon>0, \exists N \in \mathbb{N}: \quad \forall n \geqslant N, \exists y \in \mathbb{K}_{n}:|y-x|<\varepsilon .
$$

Основным резултатом работы является приводимая ниже теорема. Для ее формулировки введем обозначения $\mathrm{Q}=\operatorname{Law} S_{T}$,

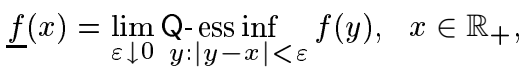

$$
\begin{aligned}
& \bar{f}(x)=\lim _{\varepsilon \downarrow 0} \text { Q-ess } \sup _{y:|y-x|<\varepsilon} f(y), \quad x \in \mathbb{R}_{+},
\end{aligned}
$$

где Q- ess inf (соответственно Q- ess sup) - существенная нижняя (соответственно верхняя) грань по мере Q. (Функции $\underline{f}$ и $\bar{f}$ корректно заданы на носителе меры $\mathrm{Q}$, а вне этого множества продолжаем их произвольно, например, доопределяем нулем.)

Теорема. Пусть функция ч удовлетворяет условиям (a)-(d), выполнено условие (2), $f$ ограничена снизу и существует а $>0$ такое, что $f\left(S_{T}\right) \leqslant a+a S_{T}$ P-n.н. Тогда

$$
I_{n}(F) \underset{n \rightarrow \infty}{\longrightarrow}\left[\int_{\mathbb{R}_{+}} \underline{f}(x) \varphi^{\prime \prime}(d x), \int_{\mathbb{R}_{+}} \bar{f}(x) \varphi^{\prime \prime}(d x)\right]
$$

в том смисле, что левье концы $I_{n}(F)$ сходятся $\kappa \int_{\mathbb{R}_{+}} \underline{f}(x) \varphi^{\prime \prime}(d x)$, а правые концы $I_{n}(F)$ сходятся $\kappa \int_{\mathbb{R}_{+}} \bar{f}(x) \varphi^{\prime \prime}(d x)$.

Из этой теоремы можно вьвести

СледствиЕ. В предположениях теоремы следующие условия әквивалентны:

(a) $I_{n}(F) \underset{n \rightarrow \infty}{\longrightarrow} I(F)$;

(b) $f$ является Q-существенно непрерывной, т.е. $f=\bar{f}$ Q-n.н.;

(c) $f$ имеет Q-n.в. непрерывную модификацию, т.е. существует функция $g$ такая, что $g=f \mathrm{Q}-$-н.н. и $g$ непрерьвна Q-n.н.

\section{СПИСОК ЛИТЕРАТУРЫ}

[1] R. Banz, M. Miller // J. Bus. 1978. V. 51. P. 653-672. [2] D. T. Breeden, R. H. Litzenberger // J. Bus. 1978. V. 51. № 4. P. 621-651. [3] J. Jackwerth // J. Derivatives. 1999. V. 7. № 2. P. 66-82. [4] A. S. Cherny. General arbitrage pricing model: probability and possibility approaches // Preprint, 2004; http://mech.math.msu.su/ cherny. [5] А. Н. Ширяев. Основы стохастической финансовой математики. М.: Фазис, 1998.

Московский государственньй университет им. М. В. Ломоносова
Представлено А. В. Булинским Принято редколлегией 25.01.2005 